\title{
MODELOS DE AFILAMENTO PARA Pinus elliottii EM DIFERENTES IDADES, NA REGIÃO DE CAÇADOR, SC
}

\author{
Saulo Jorge Téo ${ }^{1}$, Alan Marcon², Tiago Ehlers² ${ }^{2}$ Júlio César Bianchi ${ }^{2}$, Adriano Peloso ${ }^{2}$, \\ Paulo Roberto Nava ${ }^{2}$, Reinaldo Hoinacki da Costa ${ }^{3}$ \\ ${ }^{1}$ Eng. Florestal, M.Sc., Doutorando em Eng. Florestal da UFPR, Curitiba, PR, Brasil - sauloteo@ yahoo.com.br \\ ${ }^{2}$ Acadêmico de Engenharia Florestal, UNOESC, Xanxerê, SC, Brasil - marcon.alan@gmail.com; ehlers6@gmail.com; \\ julio_xxe@hotmail.com; adrianopelosobel@yahoo.com.br; paulorobertonava@hotmail.com \\ ${ }^{3}$ Eng. Florestal, Juliana Florestal Ltda., Caçador, SC, Brasil - reinaldo@ frameport.com.br \\ Recebido para publicação: 21/12/2012 - Aceito para publicação: 19/08/2013
}

\begin{abstract}
Resumo
Os objetivos deste trabalho foram ajustar e selecionar modelos de afilamento para estimar diâmetros a várias alturas e volume total, com e sem casca, de árvores de Pinus elliottii Engelm., na região de Caçador, SC, para estudar a forma dos fustes das árvores de diferentes idades. Ao todo, 78 árvores, com idades de 10, 18 e 27 anos, foram abatidas, cubadas rigorosamente e tiveram os seus volumes obtidos por meio do método de Smalian. Os modelos de afilamento testados foram os Polinômios de $2^{\circ}$ e de $5^{\circ}$ Graus e o Polinômio de Potências Fracionárias de Hradetzky. A função de afilamento que apresentou melhor desempenho para estimar o diâmetro com e sem casca ao longo do fuste das árvores foram baseadas no Polinômio de Potências Fracionárias de Hradetzky, para as idades de 10, 18 e 27 anos. O Polinômio de $2^{\circ}$ Grau propiciou as melhores estimativas de volume total com casca para as idades de 10 e 27 anos e de volume total sem casca para as idades de 18 e 27 anos. Já o Polinômio de Potências Fracionárias apresentou melhores estimativas de volume com casca para 18 anos e de volume sem casca para árvores de 10 anos. Os fustes das árvores de Pinus elliottii apresentam melhor forma nas idades mais avançadas.
\end{abstract}

Palavras-chave: Modelos polinomiais; modelos de perfil; forma do fuste.

\begin{abstract}
Taper models for Pinus elliottii, at different ages, in the region of Caçador - SC. The research aims to fit and select taper models in order to estimate diameters at different heights and total volumes, inside and outside bark, of Pinus elliottii Engelm. trees, in the region of Caçador - SC, Brazil, as well as to analyze behavior of stem form for trees at different ages. Altogether, 78 trees at 10, 18 and 27 years of age, had been cut and their volumes obtained by Smalian method. The taper models tested were the 2nd and 5th Degree Polynomial and the Hradetzky Polynomial of Fractioned Potencies. The best performance taper function for estimate diameters inside and outside bark throughout the stem were based on the Hradetzky Polynomial of Fractioned Potencies, for 10, 18 and 27 years of age. The 2nd Degree Polynomial provided the best estimates of outside bark volume for 10 and 27 years of age, and of inside bark volume for 18 and 27 years of age, although the Hradetzky Polynomial presented the best estimates of outside bark volume for 18 years of age and of inside bark volume for 10 years old trees. The stems of the Pinus elliottii trees present better form for the oldest tress.

Keywords: Polynomial models; profile models; stem form.
\end{abstract}

\section{INTRODUÇÃO}

O manejo florestal pode ser definido como a ordenação dos povoamentos para uma produção contínua de bens e serviços advindos da floresta. Para a correta aplicação e funcionalidade do manejo, as atividades desenvolvidas devem ocorrer de maneira a proporcionar o máximo de efetividade e baixos custos (SCHNEIDER; SCHNEIDER, 2008).

Segundo Scolforo (1993), à medida que a madeira se torna um componente mais importante da composição de custos de um empreendimento florestal, deve-se fazer o uso dos modelos de afilamento, pois eles são capazes de identificar as diferentes potencialidades que a árvore oferece. 
Conforme Prodan et al. (1997), os modelos de afilamento são funções que representam a variação diamétrica ao longo do fuste das árvores. Essas funções, ou modelos de afilamento, demonstram ser importante ferramenta estatística para uso frequente na estimação do volume total da árvore, ou de partes do volume total.

Os modelos de afilamento apresentam vantagens para estimar o volume de madeira por sortimento, pois são capazes de obter o volume das árvores de maneira independente nas distintas porções do fuste a serem avaliadas. Quando se usa um modelo de afilamento, utiliza-se apenas um modelo que represente o perfil médio do fuste das árvores de um povoamento, sendo ele suficiente para a estimativa de todos os tipos de volumes requeridos, como volume para laminação, laminação especial, serraria, celulose e energia.

Quando disponíveis os volumes de sortimentos de um povoamento florestal, é possível planejar a venda desse produto de forma que atinja maior valoração de mercado, maximizando os lucros. Segundo Assis (2000), os modelos de afilamento permitem que o sortimento ofereça um planejamento de produção e de logística de transporte e comercialização em função do número de toras destinadas a cada produto.

Os modelos de afilamento são um instrumento poderoso, pois podem avaliar um povoamento florestal do ponto de vista biológico, silvicultural e econômico, uma vez que permitem a descrição da forma das árvores, suas mudanças de acordo com diferentes tratos silviculturais e a valoração, de maneira minuciosa, de quanto retorno financeiro trará o povoamento florestal (FISCHER et al., 2001).

Quanto às diferentes maneiras de se medir a forma de uma árvore ou tora, é possível afirmar que não se pode medi-la como se faz com o diâmetro ou altura, apenas podem-se estabelecer parâmetros ou índices que a representam. A captação da forma tem sido feita por meio da medição de diâmetros em distintas alturas, e expressões como fatores de forma, quocientes de forma, séries de afilamento e modelos de afilamento são utilizados para defini-la (PRODAN et al., 1997).

Os fustes de hábito excurrente, como do Pinus elliottii Engelm., assemelham-se ao sólido de revolução chamado paraboloide. No entanto, uma análise mais detalhada mostra que é mais realístico considerar três partes do fuste, as quais podem ser comparadas a diferentes sólidos de revolução: a base do fuste corresponde ao neiloide, a porção central do fuste ao paraboloide e o topo do fuste corresponde a um paraboloide ou cone (ASSMANN, 1970; BURKHART; TOMÉ, 2012).

A forma é um dos mais importantes parâmetros externos de qualidade do fuste, frequentemente utilizado como critério de sortimento florestal. Fustes com forma inferior, tais como apresentando afilamento e curvatura acentuados, são usualmente relacionados com baixo rendimento em serrarias, propriedades mecânicas da madeira ruins e alto custo de processamento (TONG; ZHANG, 2008).

Na literatura florestal, é possível encontrar diversos trabalhos sobre ajuste e precisão de modelos de afilamento, sortimento florestal e estudo da forma de árvores. Para a espécie Pinus elliottii, podem-se citar os trabalhos de Scolforo et al. (1998), Drescher et al. (1999) e Floriano (2004).

Os objetivos desta pesquisa foram ajustar e selecionar modelos de afilamento para estimativa de diâmetros em diferentes alturas e volume total com e sem casca, de árvores de Pinus elliottii, na região de Caçador, SC, para estudar o comportamento da forma dos fustes de árvores de diferentes idades.

\section{MATERIAL E MÉTODOS}

Os dados foram coletados em áreas da empresa Juliana Florestal Ltda., localizadas na região de Caçador, Santa Catarina. Os povoamentos de Pinus elliottii utilizados para a realização deste estudo são provenientes dos municípios de Caçador, Macieira, Calmon, Timbó Grande, Lebon Régis, Rio das Antas e Santa Cecília.

Segundo Prates et al. (1989), a região de Caçador apresenta o clima Cfb (clima subtropical com verões brandos) da classificação de Köppen. A temperatura média anual varia de 14 a $16{ }^{\circ} \mathrm{C}$, a precipitação pluviométrica total anual é de 1300 a $2000 \mathrm{~mm}$ e a umidade relativa do ar média é de 78,1 a 82,9\%. Quanto à vegetação, originalmente ocorriam a Floresta Ombrófila Mista e Campos do Planalto. As classes predominantes de solos da região dos povoamentos em estudo são Latossolos e Cambissolos.

Ao todo, 78 árvores, com idades de 10, 18 e 27 anos, foram abatidas e submetidas à cubagem rigorosa para obtenção de seus volumes. A Tabela 1 contém a distribuição das árvores de Pinus elliottii cubadas, nas diferentes idades, bem como os valores de diâmetro médio $(d)$, diâmetro máximo $\left(d_{\text {máx }}\right)$, diâmetro mínimo $\left(d_{m i ́ n}\right)$, altura média $(h)$, altura máxima $\left(h_{m a ́ x}\right)$ e altura mínima $\left(h_{m i ́ n}\right)$. 
Foi utilizado o método de Smalian para medição do volume das árvores, em que as posições de medição do diâmetro ao longo do fuste foram definidas de maneira relativa, com base na altura total da árvore, sem considerar a altura de toco. Dessa maneira, foram medidos os diâmetros a $0,0 \%, 0,5 \%, 1 \%$, $5 \%, 10 \%, 15 \%, 20 \%, 25 \%, 30 \%, 40 \%, 50 \%, 60 \%, 70 \%, 80 \%, 90 \%$ e $95 \%$ da altura total da árvore. $\mathrm{O}$ cálculo dos volumes de cada seção foi realizado utilizando-se a fórmula de Smalian, e o volume da ponta foi obtido por meio da fórmula do volume do cone, conforme descrito em Machado e Figueiredo Filho (2009).

Tabela 1. Diâmetro, altura e número de árvores de Pinus elliottii cubadas por idade.

Table 1. Diameter, height and number of Pinus elliottii trees measured by age.

\begin{tabular}{lccccccc}
\hline $\begin{array}{l}\text { Idade } \\
(\mathbf{a n o s})\end{array}$ & $\begin{array}{c}\mathbf{n}^{\mathbf{0}} \mathbf{d e} \\
\text { árvores }\end{array}$ & $\begin{array}{c}\boldsymbol{d} \\
(\mathbf{c m})\end{array}$ & $\begin{array}{c}\boldsymbol{d}_{\text {máx }} \\
(\mathbf{c m})\end{array}$ & $\begin{array}{c}\boldsymbol{d}_{\text {mín }} \\
(\mathbf{c m})\end{array}$ & $\begin{array}{c}\boldsymbol{h} \\
(\mathbf{m})\end{array}$ & $\begin{array}{c}\boldsymbol{h}_{\text {máx }} \\
(\mathbf{m})\end{array}$ & $\begin{array}{c}\boldsymbol{h}_{\text {mín }} \\
(\mathbf{m})\end{array}$ \\
\hline 10 & 29 & 19,74 & 28,33 & 14,32 & 15,19 & 17,50 & 11,80 \\
18 & 37 & 28,07 & 48,06 & 15,92 & 23,38 & 30,20 & 17,30 \\
27 & 12 & 29,31 & 38,83 & 21,96 & 28,87 & 32,80 & 25,40 \\
\hline
\end{tabular}

O ajuste dos modelos de afilamento Polinômio de $2^{\circ}$ Grau e Polinômio de $5^{\circ}$ Grau foi realizado por meio do método dos mínimos quadrados. No entanto, o Polinômio de Potências Fracionárias exigiu o teste de diversas potências para representar o perfil do tronco da árvore, as quais, neste trabalho, foram de 0,$005 ; 0,01 ; 0,02 ; 0,03 ; 0,04 ; 0,05 ; 0,06 ; 0,07 ; 0,08 ; 0,09 ; 0,1 ; 0,2 ; 0,3 ; 0,4 ; 0,5 ; 0,6 ; 0,7 ; 0,8 ; 0,9 ; 1 ; 2 ; 3$; $4 ; 5 ; 10 ; 15 ; 20$ e 25 (Tabela 2). As potências para compor o Polinômio de Potências Fracionárias foram selecionadas com base no procedimento de regressão passo a passo, denominado backward elimination (MONTGOMERY et al., 2006), considerando um nível de significância de $10 \%(\alpha=0,1)$ no teste de "F" parcial de cada coeficiente do modelo, utilizando-se o software STATISTICA 7.

Tabela 2. Modelos de afilamento ajustados para árvores de Pinus elliottii.

Table 2. Taper functions fitted for Pinus elliottii trees.

\begin{tabular}{|c|c|c|}
\hline Denominação & Autor(es) & Modelo \\
\hline $\begin{array}{l}\text { Polinômio de } 2^{\circ} \\
\text { Grau }\end{array}$ & $\begin{array}{l}\text { Kozak et al. } \\
\qquad(1969)\end{array}$ & $\frac{d_{i j}^{2}}{d_{j}^{2}}=\beta_{0}+\beta_{1} \frac{h_{i j}}{h_{j}}+\beta_{2}{\frac{h_{i j}}{h_{j}}}^{2}$ \\
\hline $\begin{array}{l}\text { Polinômio de } 5^{\circ} \\
\text { Grau }\end{array}$ & Schöepfer (1966) & $\begin{aligned} \frac{d_{i j}}{d_{j}}=\beta_{0}+\beta_{1} \frac{h_{i j}}{h_{j}} & +\beta_{2}{\frac{h_{i j}}{h_{j}}}^{2}+\beta_{3}{\frac{h_{i j}}{h_{j}}}^{3}+\beta_{4}{\frac{h_{i j}}{h_{j}}}^{4} \\
& +\beta_{5}{\frac{h_{i j}}{h_{j}}}^{5}\end{aligned}$ \\
\hline $\begin{array}{l}\text { Polinômio de } \\
\text { Potências } \\
\text { Fracionárias }\end{array}$ & Hradetzky (1976) & $\frac{d_{i j}}{d_{j}}=\beta_{0}+\beta_{1}{\frac{h_{i j}}{h_{j}}}^{p 1}+\beta_{2}{\frac{h_{i j}}{h_{j}}}^{p 2}+\beta_{3}{\frac{h_{i j}}{h_{j}}}^{p 3}+\cdots+\beta_{n}{\frac{h_{i j}}{h_{j}}}^{p n}$ \\
\hline
\end{tabular}

$\overline{d_{i j}}$ : diâmetro na altura $h_{i j}(\mathrm{~cm}) ; d_{j}$ : diâmetro a $1,30 \mathrm{~m}$ do solo da j-ésima árvore $(\mathrm{cm}) ; h_{i j}$ : altura na posição $i$ do fuste da árvore $j$ $(\mathrm{m}) ; h_{j}$ : altura total da árvore $j(\mathrm{~m}) ; \beta_{0}, \beta_{1}, \beta_{2}, \ldots, \beta_{n}$ : coeficientes a serem estimados; $p_{0}, p_{1}, p_{2}, \ldots, p_{n}$ : potências a serem selecionadas para compor o Polinômio de Potências Fracionárias de Hradetzky.

Fonte: Scolforo (1993); Prodan et al. (1997); Scolforo et al. (1998).

O desempenho dos modelos de afilamento foi analisado de acordo com as seguintes estatísticas de ajuste e precisão: coeficiente de determinação ajustado $\left(R_{a j .}^{2}\right)$, erro padrão relativo $(s y x \%)$ e análise gráfica dos resíduos em porcentagem, conforme descrito por Montgomery et al. (2006). Além dessas estatísticas, para avaliar a acurácia das estimativas dos diâmetros com e sem casca ao longo do fuste das árvores, realizadas com os modelos de afilamento, foram calculadas as estatísticas Viés $(V)$, Média das Diferenças $(M D)$ e Desvio Padrão das Diferenças $(D P D)$, da seguinte forma:

$$
V=\frac{{\stackrel{n}{i=1} Y_{i}-{ }_{i=1}^{n} Y_{i}}_{n}}{n}
$$

$D P D=\frac{\overline{n_{i=1}^{n} Y_{i}-Y_{i}{ }^{2}-\frac{\prod_{i=1}^{n} Y_{i}-Y_{i}^{2}}{n}}}{n-p}$ 
Em que: $\quad Y_{i}=$ variável observada;

$Y_{i}=$ variável estimada;

$n=$ número de observações;

$p=$ número de coeficientes do modelo.

As estatísticas Viés $(V)$, a Média das Diferenças $(M D)$ e o Desvio Padrão das Diferenças $(D P D)$ foram calculados para as seguintes posições relativas da altura das árvores: $0,5 \%, 1 \%, 5 \%, 10 \%, 15 \%$, $20 \%, 25 \%, 30 \%, 40 \%, 50 \%, 60 \%, 70 \%, 80 \%, 90 \%$ e $95 \%$. De posse dos valores de $V, M D$ e $D P D$, o modelo de afilamento de melhor desempenho para estimativa de diâmetro com e sem casca foi selecionado para cada altura relativa, nas idades de 10, 18 e 27 anos.

Como objetivou-se estudar a precisão dos modelos de afilamento para estimativa de diâmetros com e sem casca em diferentes alturas das árvores e volume total com e sem casca, foram calculadas as estatísticas de ajuste e precisão, bem como foi realizada a análise gráfica de resíduos para essas variáveis. Os gráficos de resíduos das estimativas de diâmetro, tanto com como sem casca, foram construídos sobre as alturas relativas $\left(h_{i} h\right)$, visando analisar as estimativas ao longo do tronco das árvores de Pinus elliottii. Já os gráficos de resíduos para as estimativas de volume, com e sem casca, foram construídos sobre a variável volume estimado $\left(\mathrm{m}^{3}\right)$. Obviamente, a análise gráfica de resíduos foi feita para todas as funções de afilamento ajustadas, contudo, devido a limitações de espaço, optou-se por apresentar o gráfico de resíduos somente para as funções de afilamento de melhor desempenho. A expressão para estimativa dos diâmetros em diferentes alturas pode ser facilmente encontrada isolando-se a variável " $d_{i j}$ " nos modelos descritos na tabela 2 . Já a expressão para a estimativa dos volumes totais das árvores pode ser obtida pela integral desses modelos.

De posse da função de afilamento de melhor desempenho para as árvores de Pinus elliottii em cada idade, foi possível construir o gráfico do perfil do tronco médio para essas árvores e, por meio dele, analisar a forma dos seus fustes.

\section{RESULTADOS E DISCUSSÃO}

A tabela 3 apresenta os coeficientes e as estatísticas de ajuste e precisão das funções de afilamento ajustadas para estimar diâmetros e volume total com casca dos fustes das árvores de Pinus elliottii com idades de 10, 18 e 27 anos, na região de Caçador, SC.

Quando foram comparados os valores das estatísticas de ajuste e precisão para as funções de afilamento testadas, em todas as idades, verificou-se uma ligeira vantagem do Polinômio de Potências Fracionárias sobre o Polinômio de $5^{\circ}$ Grau, ou seja, valores de $R_{a j}^{2}$. ligeiramente mais altos e valores de $s y x \%$ ligeiramente mais baixos para as estimativas de diâmetros com casca. Por outro lado, o Polinômio de $2^{\circ}$ Grau apresentou estatísticas de ajuste e precisão inferiores às demais funções para estimativas de diâmetros com casca em todas as idades analisadas (Tabela 3).

Drescher et al. (1999) ajustaram 18 funções de afilamento, entre elas o Polinômio de $2^{\circ}$ Grau e de $5^{\circ}$ Grau, também para Pinus elliottii, em Cachoeira do Sul, RS. Entre todas as funções de afilamento testadas, o Polinômio de $5^{\circ}$ Grau apresentou o valor mais alto para $R_{a j .}^{2}(0,9770)$ e mais baixo para $s y x \%$ $(5,89)$, para estimativa do diâmetro com casca do tronco das árvores.

Considerando $R_{a j .}^{2}$, syx\% e análise gráfica de resíduos, verificou-se que o Polinômio de $2^{\circ}$ Grau apresentou desempenho superior para estimativas de volume total com casca, de árvores de Pinus elliottii com 10 e 27 anos de idade. Já para as árvores com idade de 18 anos, o Polinômio de Potências Fracionárias de Hradetzky apresentou melhor desempenho (Tabela 3 e Figura 1).

Para as idades de 10 e 27 anos, o Polinômio de $2^{\circ}$ Grau sempre apresentou valores mais altos de $R_{a j .}^{2}$ e valores mais baixos para $s y x \%$, calculados para estimativa de volume total com casca, seguido do Polinômio de Potências Fracionárias. Já o Polinômio de $5^{\circ}$ Grau apresentou os piores resultados para todas as estatísticas de ajuste e precisão para estimativa de volume total com casca (Tabela 3 ). 
Tabela 3. Ajuste e precisão das funções de afilamento para diâmetros e volumes com casca de Pinus elliottii por idade.

Table 3. Fitting and precision of taper functions for outside bark diameters and volumes of Pinus elliottii by age.

\begin{tabular}{|c|c|c|c|c|c|c|c|c|c|c|c|}
\hline \multirow{2}{*}{$\begin{array}{l}\text { Idade } \\
\text { (anos) }\end{array}$} & \multirow{2}{*}{$\begin{array}{l}\text { Função de } \\
\text { afilamento }\end{array}$} & \multirow{2}{*}{\multicolumn{6}{|c|}{ Coeficientes }} & \multicolumn{2}{|c|}{$d_{c / c}$} & \multicolumn{2}{|c|}{$v_{c / c}$} \\
\hline & & & & & & & & $R_{a j}^{2}$ & syx $\%$ & $R_{a j}^{2}$ & syx $\%$ \\
\hline \multirow[t]{4}{*}{10} & $\begin{array}{c}\text { Kozak et al. } \\
\text { (1969) }\end{array}$ & $\begin{array}{c}\mathrm{b}_{0} \\
1,309\end{array}$ & $\begin{array}{c}b_{1} \\
-2,149\end{array}$ & $\begin{array}{c}\mathrm{b}_{2} \\
0,845\end{array}$ & & & & 0,9729 & 9,13 & 0,9254 & 10,55 \\
\hline & Schöepfer & $\mathrm{b}_{0}$ & $\mathrm{~b}_{1}$ & $\mathrm{~b}_{2}$ & $b_{3}$ & $\mathrm{~b}_{4}$ & $\mathrm{~b}_{5}$ & & & & \\
\hline & (1966) & 1,205 & $-3,125$ & 13,023 & $\stackrel{-}{5} 558$ & 26,937 & $-9,481$ & 0,9846 & 6,87 & 0,9186 & 11,01 \\
\hline & $\begin{array}{l}\text { Hradetzky } \\
\text { (1976) }\end{array}$ & $\begin{array}{c}\mathrm{b}_{0} \\
1,313\end{array}$ & $\begin{array}{c}b_{0,5} \\
-1,688\end{array}$ & $\begin{array}{c}\mathrm{b}_{0,9} \\
1,805\end{array}$ & $\begin{array}{c}b_{2} \\
-1,500\end{array}$ & $\begin{array}{c}\mathrm{b}_{10} \\
0,293\end{array}$ & $\begin{array}{c}b_{15} \\
-0,221\end{array}$ & 0,9854 & 6,70 & 0,9235 & 10,68 \\
\hline \multirow[t]{5}{*}{18} & $\begin{array}{c}\text { Kozak et al. } \\
\text { (1969) }\end{array}$ & $\begin{array}{c}\mathrm{b}_{0} \\
1,281\end{array}$ & $\begin{array}{c}b_{1} \\
-2,187\end{array}$ & $\begin{array}{c}\mathrm{b}_{2} \\
0,932\end{array}$ & & & & 0,9504 & 13,08 & 0,8831 & 19,76 \\
\hline & Schöepfer & $\mathrm{b}_{0}$ & $\mathrm{~b}_{1}$ & $\mathrm{~b}_{2}$ & $\mathrm{~b}_{3}$ & $\mathrm{~b}_{4}$ & $\mathrm{~b}_{5}$ & & & & \\
\hline & (1966) & 1,220 & $-3,993$ & 17,678 & $\begin{array}{c}- \\
38,507\end{array}$ & 36,692 & $\begin{array}{c}- \\
13,097\end{array}$ & 0,9803 & 8,24 & 0,8716 & 20,71 \\
\hline & Hradetzky & $\mathrm{b}_{0}$ & $\mathrm{~b}_{0,5}$ & $\mathrm{~b}_{0,9}$ & $\mathrm{~b}_{1}$ & $b_{10}$ & & & & & \\
\hline & (1976) & 1,470 & $-4,777$ & 25,156 & $21, \overline{748}$ & $-0,103$ & & 0,9823 & 7,82 & 0,8865 & 19,47 \\
\hline \multirow[t]{4}{*}{27} & $\begin{array}{l}\text { Kozak et al. } \\
\quad \text { (1969) }\end{array}$ & $\begin{array}{c}\mathrm{b}_{0} \\
1,212\end{array}$ & $\begin{array}{c}b_{1} \\
-1,790\end{array}$ & $\begin{array}{c}\mathrm{b}_{2} \\
0,597\end{array}$ & & & & 0,9530 & 11,40 & 0,9389 & 10,77 \\
\hline & Schöepfer & $\mathrm{b}_{0}$ & & & $\mathrm{~b}_{3}$ & & $\mathrm{~b}_{5}$ & 0,9847 & 6,49 & 0,9057 & 13,38 \\
\hline & (1966) & 1,181 & $-3,424$ & 14,621 & 30,802 & 28,613 & 10,194 & & & & \\
\hline & $\begin{array}{l}\text { Hradetzky } \\
\text { (1976) }\end{array}$ & $\begin{array}{c}\mathrm{b}_{0} \\
1,342\end{array}$ & $\begin{array}{c}\mathrm{b}_{0,4} \\
-1,501\end{array}$ & $\begin{array}{c}\mathrm{b}_{0,7} \\
1,045\end{array}$ & $\begin{array}{c}b_{3} \\
-0,879\end{array}$ & $\begin{array}{c}b_{25} \\
-0,005\end{array}$ & & 0,9867 & 6,06 & 0,9239 & 12,02 \\
\hline
\end{tabular}

Para a idade de 18 anos, o Polinômio de Potências Fracionárias sempre apresentou valores mais altos para $R_{a j}^{2}$. e valores mais baixos para $s y x \%$, seguido do Polinômio de $2^{\circ}$ Grau e, por fim, do Polinômio de $5^{\circ}$ Grau (Tabela 3 ).

Scolforo et al. (1998) estudaram a precisão de diversas equações de afilamento para estimativa de volume total de Pinus elliottii, dentre as quais o Polinômio de Potências Fracionárias e o Polinômio de $5^{\circ}$ Grau, que apresentaram valores de $R^{2}$ entre 0,97 e 0,99 e $s y x \%$ entre 5,36 e 7,91, com ligeira superioridade para o Polinômio de Potências Fracionárias.

De acordo com Eisfeld et al. (2004), tanto o Polinômio de Potências Fracionárias como o Polinômio de $5^{\circ}$ Grau proporcionaram boas estimativas para o volume total de Pinus taeda L., em Rio Negro, PR, apresentando valores de $R_{a j}^{2}$. acima de 0,94 e de $s y x \%$ abaixo de $10 \%$, com leve superioridade do Polinômio de Potências Fracionárias.

Quando foram analisadas as estatísticas $V$ e $M D$, tornou-se evidente a superioridade das estimativas de diâmetro com casca do Polinômio de Potências Fracionárias e do Polinômio de $5^{\circ}$ Grau sobre o Polinômio de $2^{\circ}$ Grau. Este último sempre apresentou valores mais acentuados para $V$ e $M D$, com poucas exceções, como para a altura relativa de $30 \%$ para árvores de 10 anos e de $80 \%$ para as árvores de 18 e 27 anos.

Souza et al. (2008) compararam diferentes modelos de afilamento para Pinus taeda em Campo Belo do Sul, SC, e recomendaram que os valores de $V$ não devem exceder aos limites de \pm 1 . Os valores de $V$ do Polinômio de Potências Fracionárias não ultrapassaram \pm 1 para todas as alturas relativas e idades avaliadas. Para o Polinômio de $5^{\circ} \mathrm{Grau}$, foram observados valores de $-1,39$ e $-1,61$ na altura relativa de $5 \%$, para as idades de 18 e 27 anos, respectivamente. No entanto, para o Polinômio de $2^{\circ} \mathrm{Grau}$, os valores de $V$ excedem ao limite de \pm 1 em diversas alturas relativas, principalmente nas posições mais inferiores e superiores do fuste. Quanto à estatística $D P D$, os resultados foram mais próximos para as três funções de afilamento testadas. De maneira geral, para as idades de 10, 18 e 27 anos, o Polinômio de Potências 
Fracionárias apresentou valores mais baixos de $D P D$ para as alturas relativas entre 5 e $20 \%$ e acima de $90 \%$, enquanto que o Polinômio de $2^{\circ}$ Grau, para as alturas relativas entre 25 e $80 \%$.

Dist. de Resíduos (Hradetzky) - 10

anos

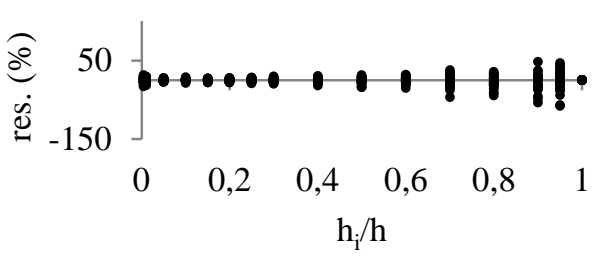

Dist. de Resíduos (Hradetzky) - 18

anos

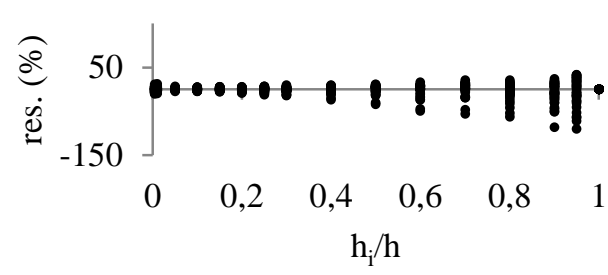

Dist. de Resíduos (Hradetzky) - 27

anos

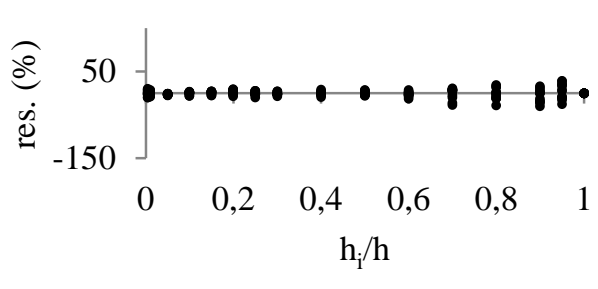

Dist. Resíduos (Kozak) - 10 anos

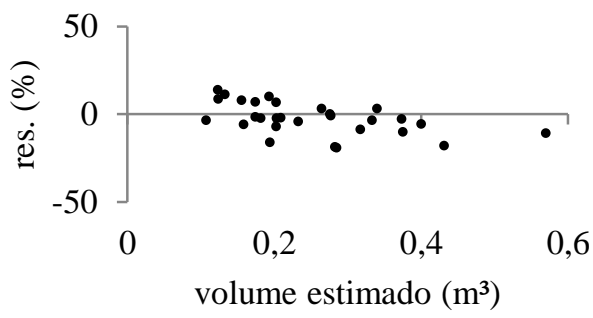

Dist. Resíduos (Hradetzky) - 18

anos

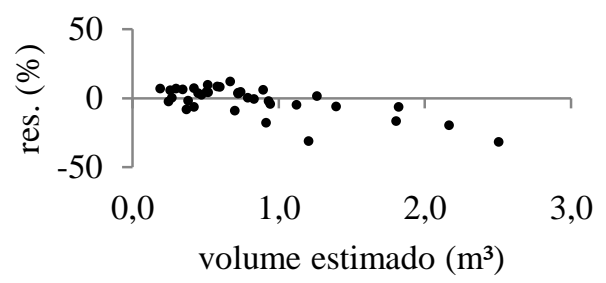

Dist. Resíduos (Kozak) - 27 anos

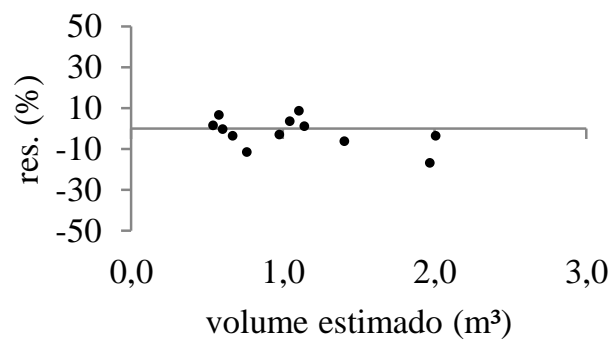

Figura 1. Distribuição de resíduos das funções de afilamento de melhor desempenho, para estimativa de diâmetros e volume total com casca de árvores de Pinus elliottii por idade.

Figure 1. Residue distribution of the best performance taper function, for estimate diameter and total volume outside bark of Pinus elliottii trees, by age.

No entanto, quando consideradas as estatísticas $V, M D$ e $D P D$ de maneira conjunta, verifica-se que o Polinômio de Potências Fracionárias derivou as estimativas de diâmetro com casca mais acuradas para a maioria das alturas relativas do fuste de Pinus elliottii, em todas as idades estudadas (Tabela 5).

Na figura 1 estão contidos os gráficos da distribuição de resíduos para as funções de afilamento de melhor desempenho, para estimativa de diâmetros e volume total com casca de árvores de Pinus elliottii, com idades de 10, 18 e 27 anos, na região de Caçador, SC.

Observando-se os gráficos de dispersão de resíduos para as funções de afilamento de melhor desempenho para estimar o diâmetro com casca (Figura 1), nota-se que a amplitude dos valores de resíduos aumenta a partir da posição de $70 \%$ da altura total das árvores, exceto para as árvores de 27 anos de idade. Adicionalmente, pode-se observar uma tendência de superestimativa dos diâmetros com casca na porção superior do fuste das árvores para o Polinômio de Potências Fracionárias, ajustado para a idade de 18 anos.

Os gráficos de resíduos resultantes dos ajustes do Polinômio de Potências Fracionárias e do Polinômio de $5^{\circ}$ Grau para estimativa do diâmetro com casca foram muito semelhantes para todas as 
idades analisadas. No entanto, o Polinômio de $2^{\circ}$ Grau apresentou tendências muito acentuadas, principalmente em superestimar os diâmetros com casca da porção superior do fuste das árvores de Pinus elliottii. Essa tendenciosidade também foi evidenciada pelos valores de $V$ do Polinômio de $2^{\circ} \mathrm{Grau}$, que foram de -1,12 a -2,81 para as alturas relativas de 90 e $95 \%$.

Quanto à dispersão de resíduos para estimativa de volume total com casca, observa-se uma tendenciosidade de superestimativa do volume das maiores árvores. Essa tendência é mais acentuada para a idade de 18 anos, seguida das idades de 10 e, por fim, 27 anos. No entanto, quando analisados os resíduos para as menores árvores, não são identificadas tendências de super ou subestimativas, com valores distribuídos de maneira uniforme. Convém salientar que a dispersão de resíduos de todas as funções de afilamento testadas apresentou-se de maneira muito semelhante, quando analisadas para as idades de 10, 18 e 27 anos (Figura 1).

A tabela 4 apresenta os coeficientes e as estatísticas de ajuste e precisão das funções de afilamento para estimar diâmetros e volume total sem casca, dos fustes das árvores de Pinus elliottii com idades de 10, 18 e 27 anos, na região de Caçador, SC.

Tabela 4. Ajuste e precisão das funções de afilamento para diâmetros e volumes sem casca de Pinus elliottii por idade.

Table 4. Fitting and precision the taper functions for inside bark diameters and volumes of Pinus elliottii by age.

\begin{tabular}{|c|c|c|c|c|c|c|c|c|c|c|c|c|}
\hline \multirow{2}{*}{$\begin{array}{l}\text { Idade } \\
(\text { anos })\end{array}$} & \multirow{2}{*}{$\begin{array}{l}\text { Função de } \\
\text { afilamento }\end{array}$} & \multirow{2}{*}{\multicolumn{7}{|c|}{ Coeficientes }} & \multicolumn{2}{|c|}{$d_{s / c}$} & \multicolumn{2}{|c|}{$v_{s / c}$} \\
\hline & & & & & & & & & $K_{a j}$ & syx\% & $a j$. & syx $\%$ \\
\hline \multirow[t]{4}{*}{10} & $\begin{array}{c}\text { Kozak et al. } \\
\text { (1969) }\end{array}$ & $\begin{array}{c}\mathrm{b}_{0} \\
0.948\end{array}$ & $\begin{array}{c}b_{1} \\
-1352\end{array}$ & $\begin{array}{c}b_{2} \\
0384\end{array}$ & & & & & 0,9753 & 8,75 & 0,9108 & 11,48 \\
\hline & Schöepfer & $\mathrm{b}_{0}$ & $\mathrm{~b}_{1}$ & $\mathrm{~b}_{2}$ & $\mathrm{~b}_{3}$ & $\mathrm{~b}_{4}$ & $\mathrm{~b}_{5}$ & & 0.9834 & 7.18 & 0.9085 & 11.63 \\
\hline & (1966) & 1,011 & $-2,154$ & 8,998 & $-19,76$ & 17,804 & & & & & & \\
\hline & $\begin{array}{c}\text { Hradetzky } \\
\text { (1976) }\end{array}$ & $\begin{array}{c}b_{0} \\
-130,0\end{array}$ & $\begin{array}{c}\mathrm{b}_{0,01} \\
158,59\end{array}$ & $\begin{array}{c}b_{0,08} \\
-29,74\end{array}$ & $\begin{array}{c}\mathrm{b}_{0,9} \\
19,563\end{array}$ & $\begin{array}{c}b_{1} \\
-18,41\end{array}$ & & & 0,9839 & 7,07 & 0,9192 & 10,93 \\
\hline \multirow[t]{5}{*}{18} & $\begin{array}{l}\text { Kozak et al. } \\
\quad(1969)\end{array}$ & $\begin{array}{c}\mathrm{b}_{0} \\
1,054\end{array}$ & $\begin{array}{c}b_{1} \\
-1,558\end{array}$ & $\begin{array}{c}b_{2} \\
0,506\end{array}$ & & & & & 0,9643 & 11,02 & 0,9148 & 17,52 \\
\hline & Schöepfer & $\mathrm{b}_{0}$ & $\mathrm{~b}_{1}$ & $\mathrm{~b}_{2}$ & $\mathrm{~b}_{3}$ & $\mathrm{~b}_{4}$ & $\mathrm{~b}_{5}$ & & 09801 & 823 & 09997 & 1803 \\
\hline & (1966) & 1,096 & $-3,232$ & 14,805 & $-32,82$ & 31,262 & $-11,12$ & & & & ועם, & \\
\hline & Hradetzky & $\mathrm{b}_{0}$ & $\mathrm{~b}_{0,5}$ & $\mathrm{~b}_{0,8}$ & $\mathrm{~b}_{0,9}$ & $b_{10}$ & $\mathrm{~b}_{15}$ & $\mathrm{~b}_{20}$ & 09820 & 783 & 09137 & 1763 \\
\hline & (1976) & 1,349 & $-6,127$ & 26,044 & $-21,09$ & $-0,950$ & 1,536 & $-0,764$ & $0,90<0$ & $1,0 J$ & ו & 17,05 \\
\hline \multirow[t]{5}{*}{27} & Kozak et al. & $\mathrm{b}_{0}$ & $\mathrm{~b}_{1}$ & $\mathrm{~b}_{2}$ & & & & & 0,9632 & 9,79 & 0,9030 & 13,08 \\
\hline & (1969) & 0,988 & $-1,193$ & 0,204 & & & & & & & & \\
\hline & Schöepfer & $\mathrm{b}_{0}$ & $\mathrm{~b}_{1}$ & $\mathrm{~b}_{2}$ & $\mathrm{~b}_{3}$ & $\mathrm{~b}_{4}$ & $\mathrm{~b}_{5}$ & & 0,9801 & 7,21 & 0,8569 & 15,89 \\
\hline & (1966) & 1,054 & $-2,639$ & 11,591 & $-24,83$ & 23,045 & $-8,224$ & & & & & \\
\hline & $\begin{array}{c}\text { Hradetzky } \\
\text { (1976) }\end{array}$ & $\begin{array}{c}b_{0} \\
1,181\end{array}$ & $\begin{array}{c}b_{0,4} \\
-1,185\end{array}$ & $\begin{array}{c}\mathrm{b}_{0,7} \\
0,865\end{array}$ & $\begin{array}{c}b_{3} \\
-0,860\end{array}$ & $\begin{array}{c}\mathrm{b}_{20} \\
0,002\end{array}$ & & & 0,9819 & 6,88 & 0,8822 & 14,42 \\
\hline
\end{tabular}

$d_{s / c}$ : diâmetro sem casca; $v_{s / c}$ : volume total sem casca; $R_{a j}^{2}:$ coeficiente de determinação ajustado; syx\%: erro padrão relativo (\%).

Da mesma maneira que ocorreu para a estimativa dos diâmetros com casca, agora, para estimativa dos diâmetros sem casca, o Polinômio de Potências Fracionárias de Hradetzky sempre apresentou valores mais altos para $R_{a j}^{2}$. e valores mais baixos de $s y x \%$, seguido do Polinômio de $5^{\circ}$ Grau, para todas as idades analisadas. Por sua vez, o Polinômio de $2^{\circ}$ Grau apresentou valores inferiores para as estatísticas de ajuste e precisão quanto à estimativa do diâmetro sem casca, em todas as idades estudadas (Tabela 4).

Considerando as estatísticas de ajuste e precisão e análise gráfica de resíduos, verificou-se que o Polinômio de $2^{\circ}$ Grau apresentou desempenho superior para estimativas de volume total sem casca de árvores de Pinus elliottii com 18 e 27 anos de idade. Já para as árvores com idade de 10 anos, o Polinômio de Potências Fracionárias de Hradetzky apresentou melhor desempenho (Tabela 4 e Figura 2).

Para as idades de 18 e 27 anos, o Polinômio de $2^{\circ}$ Grau sempre apresentou valores mais altos de $R_{a j}^{2}$. e valores mais baixos para $s y x \%$, calculados para estimativa de volume total sem casca, seguido do 
Polinômio de Potências Fracionárias. Já o Polinômio de $5^{\circ}$ Grau, considerando as idades de 18 e 27 anos, sempre apresentou os piores resultados para $R_{a j}^{2}$. e $s y x \%$, para estimativa de volume total sem casca.

Para as árvores com idade de 10 anos, o Polinômio de Potências Fracionárias sempre apresentou valores mais altos para $R_{a j}^{2}$. e valores mais baixos para $s y x \%$, sendo, assim, a função de afilamento de melhor desempenho para estimativa do volume total sem casca, seguida do Polinômio de $2^{\circ}$ Grau e, por fim, do Polinômio de $5^{\circ}$ Grau.

Os valores de $V$ e $M D$ apontaram maior acuracidade das estimativas de diâmetro sem casca do Polinômio de Potências Fracionárias, acompanhado de perto pelo Polinômio de $5^{\circ}$ Grau, para a maioria das alturas relativas, em todas as idades analisadas. Por outro lado, o Polinômio de $2^{\circ}$ Grau apresentou valores mais acentuados de $V$ e $M D$ para as estimativas de diâmetro sem casca na grande maioria das alturas relativas, com raras exceções, como, por exemplo, para a posição de $25 \%$ na idade de 10 anos, $30 \%$ para árvores de 18 anos e 20\% para 27 anos. O Polinômio de Potências Fracionárias não apresentou nenhum valor de $V$ excedendo aos limites de \pm 1 , considerando todas as alturas relativas e idades estudadas, o que é desejável, conforme Souza et al. (2008). O mesmo não ocorreu para os Polinômios de $2^{\circ}$ e $5^{\circ}$ Graus. Quanto ao $D P D$, o Polinômio de $2^{\circ}$ Grau apresentou-se superior às demais funções de afilamento em várias alturas relativas, principalmente para as posições intermediárias dos fustes das árvores, entre 30 e $70 \%$, para todas as idades analisadas.

Ainda assim, quando consideradas as estatísticas $V, M D$ e $D P D$ de maneira conjunta, verifica-se que o Polinômio de Potências Fracionárias derivou as estimativas de diâmetro sem casca mais acuradas para a maioria das alturas relativas do fuste de Pinus elliottii em todas as idades estudadas (Tabela 5).

Os gráficos de resíduos para as funções de afilamento de melhor desempenho, para estimativa de diâmetros e volume total sem casca de árvores de Pinus elliottii com idades de 10, 18 e 27 anos, na região de Caçador, SC, são apresentados na figura 2.

Quanto aos gráficos de dispersão de resíduos para as funções de afilamento de melhor desempenho para estimar o diâmetro sem casca, é possível notar que a amplitude dos valores de resíduos aumenta a partir da posição de $70 \%$ da altura total das árvores, exceto para as árvores de 27 anos de idade, tal qual ocorreu para o diâmetro com casca. Adicionalmente, pode-se observar uma tendência de superestimativa dos diâmetros sem casca na porção superior do fuste das árvores, para as idades de $10 \mathrm{e}$ 18 anos, entretanto essa tendência apresenta-se de maneira mais acentuada para o Polinômio de Potências Fracionárias ajustado para a idade de 18 anos.

Da mesma forma que ocorreu para as estimativas dos diâmetros com casca, para os diâmetros sem casca os gráficos de resíduos resultantes dos ajustes do Polinômio de Potências Fracionárias e do Polinômio de $5^{\circ}$ Grau foram muito semelhantes para todas as idades analisadas. O Polinômio de $2^{\circ}$ Grau apresentou tendências muito acentuadas, principalmente em superestimar os diâmetros sem casca da porção superior do fuste das árvores de Pinus elliottii. Pode-se afirmar que essa tendência é ratificada pelos valores negativos mais acentuados de $V$ para o Polinômio de $2^{\circ}$ Grau, nas alturas relativas de 90 e 95\% (Figura 2).

A dispersão de resíduos para estimativa de volume total sem casca apresenta tendenciosidade de superestimativa para as maiores árvores. Essa tendência é mais acentuada para a idade de 18 anos, seguida das idades de $10 \mathrm{e}$, por fim, 27 anos. No entanto, quando analisados os resíduos para as menores árvores, há maior uniformidade na distribuição. Convém ressaltar que a dispersão de resíduos de todas as funções de afilamento testadas ocorreu de maneira muito semelhante, quando analisadas para as idades de 10, 18 e 27 anos (Figura 2).

Queiroz et al. (2006) e Favalessa et al. (2012b) estudaram a acuracidade de funções de afilamento não somente para estimativas de volume total, mas para o volume estimado em diferentes alturas relativas do fuste de árvores de Mimosa scabrella Bentham e Tectona grandis L. f., respectivamente. De acordo com Queiroz et al. (2006), o Polinômio de Potências Fracionárias estimou os volumes com maior acuracidade, seguido do Polinômio de $5^{\circ}$ Grau e, com os piores resultados, do Polinômio de $2^{\circ}$ Grau. Segundo Favalessa et al. (2012b), o Polinômio de $5^{\circ}$ Grau foi mais acurado para estimar volumes ao longo do fuste.

Conforme os resultados de $V, M D$ e $D P D$, foi construída a Tabela 5, a qual apresenta a função de afilamento de melhor desempenho para estimar os diâmetros com e sem casca, para as diferentes alturas relativas das árvores de Pinus elliottii, em cada idade estudada. 
Dist. de Resíduos (Hradetzky) - 10 anos

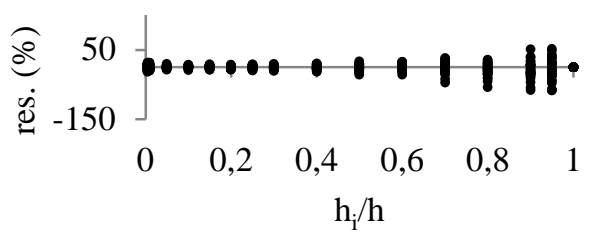

Dist. de Resíduos (Hradetzky) - 18 anos

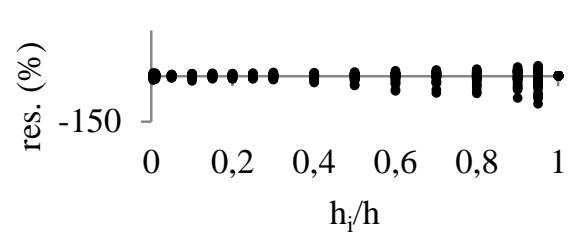

Dist. de Resíduos (Hradetzky) - 27 anos

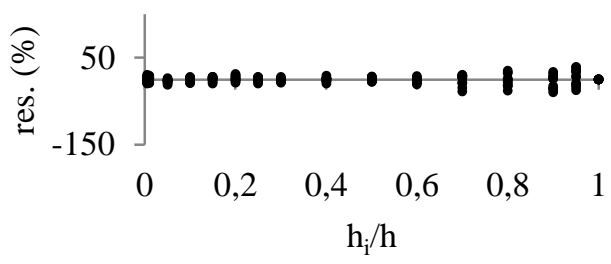

Dist. Resíduos (Hradetzky) - 10

anos

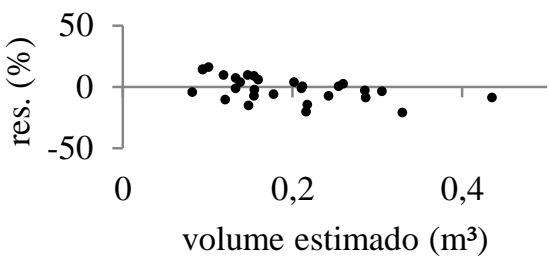

Dist. Resíduos (Kozak) - 18 anos

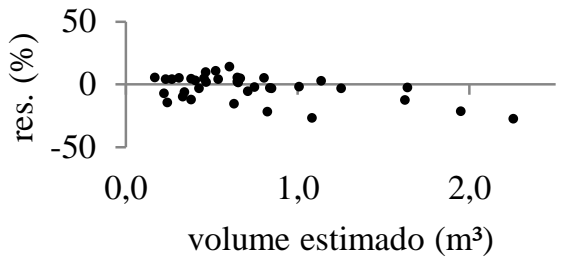

Dist. Resíduos (Kozak) - 27 anos

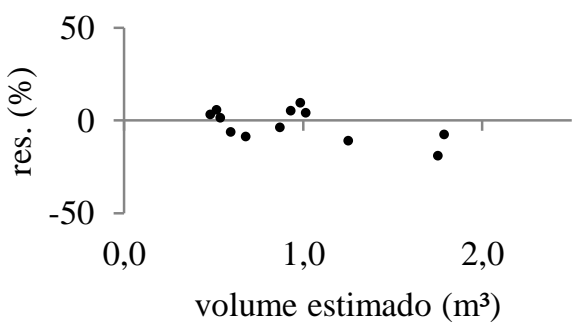

Figura 2. Distribuição de resíduos das funções de afilamento de melhor desempenho, para estimativa de diâmetros e volume total sem casca de árvores de Pinus elliottii por idade.

Figure 2. Residue distribution of the best performance taper function, for estimate diameter and total volume inside bark of Pinus elliottii trees, by age.

Pela análise da tabela 5, verifica-se que o Polinômio de Potências Fracionárias foi selecionado para estimativa de diâmetros, tanto com como sem casca, para a maioria das alturas relativas do fuste de Pinus elliottii, para as três idades estudadas. Todavia, para estimativa de diâmetros sem casca ao longo do fuste das árvores de 10 anos, tanto o Polinômio de Potências Fracionárias como o Polinômio de $5^{\circ}$ Grau foram selecionados para seis alturas relativas. Nesse caso, o Polinômio de Potências Fracionárias ainda foi considerado superior ao Polinômio de $5^{\circ}$ Grau, por apresentar-se como segundo colocado mais vezes que o Polinômio de $5^{\circ}$ Grau.

Também é possível observar na tabela 5 a superioridade do Polinômio de Potências Fracionárias para estimar os diâmetros com e sem casca nas porções inferiores $(0,5$ a $20 \%)$ e nas porções superiores (90 e 95\%) dos fustes das árvores, para todas as idades estudadas. Assis et al. (2002) e Favalessa et al. (2012a) também destacaram as estimativas de diâmetro mais acuradas para o Polinômio de Potências Fracionárias na porção inferior das árvores, atribuindo esse resultado à inclusão de variáveis de maior potência, as quais conseguem descrever melhor as irregularidades dessa parte das árvores.

Scolforo et al. (1998) estudaram a acurácia de equações de afilamento para representar o perfil do fuste de Pinus elliottii na região nordeste do Paraná. Entre as funções testadas, além do Polinômio de Potências Fracionárias e do Polinômio de $5^{\circ}$ Grau, os autores testaram a Função Spline Cúbica, o Modelo de Amateis e Burkhart e o Modelo de Clutter. De acordo com os resultados encontrados, concluiu-se que 
o Polinômio de Potências Fracionárias propiciou as estimativas mais acuradas do diâmetro ao longo do fuste das árvores, seguido do Modelo de Amateis e Burkhart e do Polinômio de $5^{\circ}$ Grau. Por outro lado, Drescher et al. (1999) testaram diversas funções de afilamento, entre as quais os Polinômios de $2^{\circ}, 3^{\circ}, 4^{\circ} \mathrm{e}$ $5^{\circ}$ Graus, para Pinus elliottii, no Rio Grande do Sul, na qual o Polinômio de $5^{\circ}$ Grau foi selecionado para descrever a forma do tronco, por apresentar boa precisão e por ter distribuição de resíduos mais uniforme.

Assis et al. (2001), Fischer et al. (2001) e Yoshitani Júnior et al. (2012) estudaram diversas funções de afilamento para representar o perfil do fuste de árvores de Pinus taeda, no estado do Paraná. Todos esses autores recomendaram o Polinômio de Potências Fracionárias para estimar os diâmetros ao longo do fuste das árvores de Pinus taeda. Além disso, Yoshitani Júnior et al. (2012) destacaram a semelhança entre as estatísticas de ajuste e precisão do Polinômio de Potências Fracionárias e do Polinômio de $5^{\circ}$ Grau.

Tabela 5. Seleção das funções de afilamento para estimativas de diâmetros com e sem casca, ao longo do fuste de árvores de Pinus elliottii com idades de 10, 18 e 27 anos.

Table 5. Selection of the taper functions for estimate diameters outside and inside bark, throughout the stem of Pinus elliottii trees, at 10,18 and 27 years of age.

\begin{tabular}{lllllll}
\hline \multirow{2}{*}{ Altura relativa (\%) } & \multicolumn{2}{c}{ Idade de 10 anos } & \multicolumn{2}{c}{ Idade de $\mathbf{1 8}$ anos } & \multicolumn{2}{c}{ Idade de 27 anos } \\
\cline { 2 - 7 } & \multicolumn{1}{c}{$\boldsymbol{c} \boldsymbol{c}$} & $\boldsymbol{d}_{\boldsymbol{s} \boldsymbol{c}}$ & $\boldsymbol{\boldsymbol { d } _ { \boldsymbol { c } \boldsymbol { c } }}$ & $\boldsymbol{\boldsymbol { d } _ { \boldsymbol { s } \boldsymbol { c } }}$ & $\boldsymbol{d}_{\boldsymbol{c} \boldsymbol{c}}$ & $\boldsymbol{d}_{\boldsymbol{s} \boldsymbol{c}}$ \\
\hline 0,5 & Hrad & Schö & Hrad & Hrad & Hrad & Hrad \\
1 & Hrad & Schö & Hrad & Schö & Hrad & Schö \\
5 & Hrad & Hrad & Hrad & Hrad & Hrad & Hrad \\
10 & Schö & Schö & Hrad & Hrad & Hrad & Hrad \\
15 & Hrad & Hrad & Hrad & Hrad & Hrad & Hrad \\
20 & Hrad & Hrad & Hrad & Hrad & Hrad & Koz \\
25 & Hrad & Koz & Schö & Hrad & Schö & Schö \\
30 & Koz & Koz & Schö & Koz & Hrad & Hrad \\
40 & Koz & Koz & Hrad & Hrad & Hrad & Hrad \\
50 & Hrad & Hrad & Hrad & Hrad & Hrad & Hrad \\
60 & Schö & Schö & Hrad & Schö & Schö & Schö \\
70 & Koz & Schö & Hrad & Hrad & Hrad & Hrad \\
80 & Hrad & Schö & Koz & Schö & Koz & Schö \\
90 & Hrad & Hrad & Hrad & Hrad & Hrad & Hrad \\
95 & Schö & Hrad & Hrad & Hrad & Schö & Schö \\
\hline
\end{tabular}

$d_{c / c}$ : diâmetro com casca; $d_{s / c}$ : diâmetro sem casca; Hrad: Hradetzky (1976), Polinômio de Potências Fracionárias; Schö: Schöepfer (1966), Polinômio de $5^{\circ}$ Grau; Koz = Kozak et al. (1969), Polinômio de $2^{\circ}$ Grau.

Machado et al. (2004), Miguel et al. (2011) e Queiroz et al. (2006) testaram o Polinômio de $2^{\circ}$ Grau, de $5^{\circ}$ Grau e de Potências Fracionárias para estimar diâmetros e volumes de Pinus oocarpa Schiede, Eucalyptus urophylla S. T. Blake e Mimosa scabrella, respectivamente. Ao final de seus estudos, esses autores concluíram que, na maioria dos casos, o Polinômio de Potências Fracionárias apresentou as melhores estimativas dos diâmetros ao longo do fuste das árvores.

A figura 3 apresenta o perfil do fuste médio das árvores de Pinus elliottii, com idades de 10, $18 \mathrm{e}$ 27 anos, obtidos pelo Polinômio de Potências Fracionárias, a qual foi a função de afilamento de melhor desempenho para a maioria das alturas relativas, para o diâmetro tanto com como sem casca (Tabela 5).

Analisando a figura 3, pode-se afirmar que a forma do fuste das árvores de Pinus elliottii foi semelhante para as idades de 18 e 27 anos, mas o mesmo não ocorre para a idade de 10 anos. Para as idades de 18 e 27 anos, o perfil do fuste das árvores apresentou uma linha convexa com relação ao eixo das ordenadas, da base até cerca de $10 \%$ da altura total, onde exibe um ponto de inflexão. Para as árvores de 10 anos, essa linha convexa estende-se até 15 a 20\% da altura total. Essa primeira porção do fuste das árvores se assemelha a um sólido geométrico do tipo neiloide.

Para o restante do fuste das árvores de 18 e 27 anos, o perfil do tronco assume forma semelhante a um paraboloide, onde o afilamento inicialmente é menos acentuado (de 10 até $40 \%$ e $60 \%$ da altura total para 18 e 27 anos, respectivamente) e passa a ser mais acentuado no topo da árvore. As árvores de 10 
anos de idade apresentam perfil do tronco semelhante a um paraboloide de 20 até $60 \%$ da altura total. A partir dessa posição, o tronco assume forma mais próxima a um cone.

Ao analisar a porção inferior dos fustes das árvores, percebe-se que há um afilamento mais acentuado para as árvores de 18 e 27 anos, podendo-se afirmar que essas árvores sofrem maior influência da dilatação da base do tronco.

Por outro lado, quando se toma como referência a porção intermediária do fuste, entre as alturas relativas de 15 a $60 \%$, o afilamento é tanto menor quanto maior a idade. Considerando-se valores médios de diâmetros ao longo do fuste, tem-se um afilamento de 1,00, 0,84 e $0,57 \mathrm{~cm} / \mathrm{m}$ entre 15 e $60 \%$ da altura relativa, para as idades de 10, 18 e 27 anos, respectivamente. Sendo assim, pode-se afirmar que as árvores de idade mais avançada apresentam menor afilamento e, consequentemente, melhor forma (Figura 3).

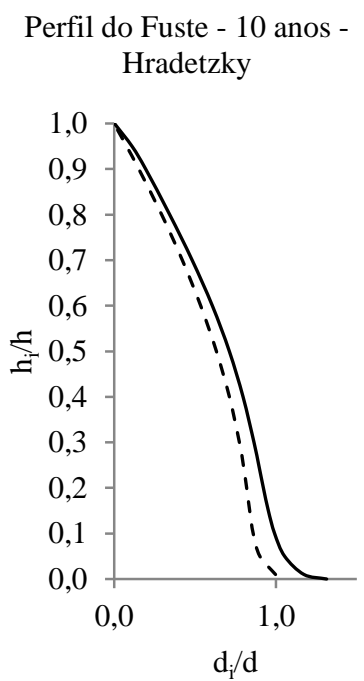

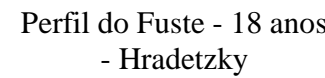

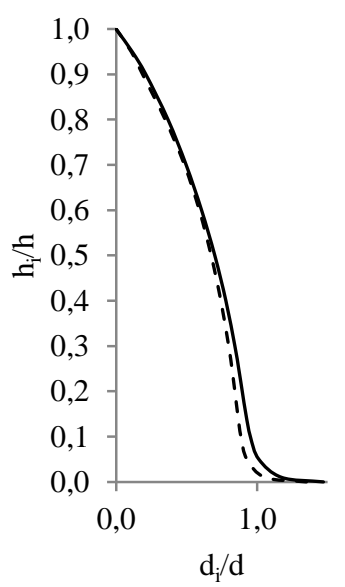

Perfil do Fuste - 27 anos Hradetzky

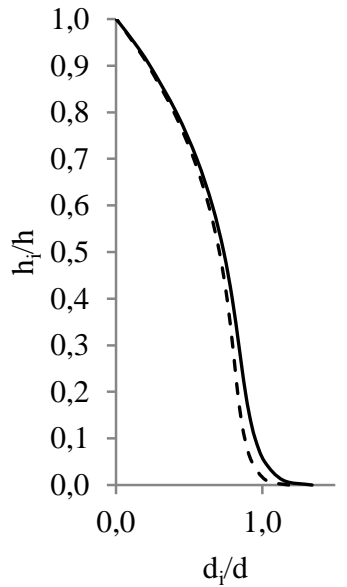

\section{Diâmetro com casca - - - Diâmetro sem casca}

Figura 3. Perfil do fuste obtido pela função de afilamento de melhor desempenho, para estimar diâmetros ao longo do fuste de Pinus elliottii por idade.

Figure 3. Stem profile obtained by the best performance taper function, for estimate diameters throughout the stem of Pinus elliottii by age.

De acordo com Queiroz (2006), de maneira geral, os fustes de Mimosa scabrella apresentam tendência de melhorar a forma com o aumento da idade, entretanto, na porção inferior do fuste ocorre o contrário: ele apresenta-se mais irregular. Essa tendência é semelhante à encontrada neste estudo, para Pinus elliottii (Figura 3).

Larson (1963) elaborou uma extensa e minuciosa revisão bibliográfica a respeito dos diferentes fatores que afetam a forma das árvores, dentre eles a idade, chegando à conclusão de que a maioria desses fatores tem estreita relação com a variável altura ou comprimento de copa. Possivelmente, a copa das árvores com 10 anos de idade ocupa maior proporção da altura total, enquanto que para as árvores de $18 \mathrm{e}$ 27 anos a copa concentra-se nas porções superiores do fuste, proporcionando um afilamento menos acentuado na porção intermediária.

Segundo Floriano (2004), a redução da altura de copa por meio de tratamentos de desrama, realizados em Pinus elliottii com 6 anos de idade, resultou em mudanças significativas na forma do fuste das árvores, de maneira que as árvores sem desrama apresentaram forma cônica, com tendência à neiloide, e as árvores com desrama apresentaram forma cônica, com tendência à paraboloide.

Friedl (1989) estudou o comportamento do fator de forma natural do tronco de árvores de Araucaria angustifolia (Bert.) O. Ktze. segundo a idade, o sítio e a posição sociológica, e os resultados mostraram que a idade foi o fator que mais provocou variação na forma da árvore. 


\section{CONCLUSÕES}

- De maneira geral, a função de afilamento mais acurada para estimar os diâmetros com e sem casca ao longo do fuste de Pinus elliottii, na região de Caçador, SC, foi o Polinômio de Potências Fracionárias de Hradetzky, para as idades de 10, 18 e 27 anos.

- Para estimativa de volume total com casca de Pinus elliottii, as funções de afilamento de melhor desempenho foram o Polinômio de $2^{\circ}$ Grau para 10 e 27 anos e o Polinômio de Potências Fracionárias de Hradetzky para 18 anos de idade.

- Para estimativa de volume total sem casca de Pinus elliottii, o Polinômio de $2^{\circ}$ Grau propiciou as melhores estimativas para 18 e 27 anos e o Polinômio de Potências Fracionárias de Hradetzky para 10 anos de idade.

- Os fustes das árvores de Pinus elliottii, na região de Caçador, SC, apresentam menor afilamento e, portanto, melhor forma, para as idades mais avançadas.

\section{REFERÊNCIAS}

ASSIS, A. L. de. Avaliação de modelos polinomiais segmentados e não segmentados na estimativa de diâmetros e volumes comerciais de Pinus taeda. 189 f. Dissertação (Mestrado em Engenharia Florestal) - Universidade Federal de Lavras, Lavras, 2000.

ASSIS, A. L. de; SCOLFORO, J. R. S.; MELLO, J. M. de; ACERBI JÚNIOR, F. W.; OLIVEIRA, A. D. de. Comparação de modelos polinomiais segmentados e não segmentados na estimativa de diâmetros e volumes ao longo do fuste de Pinus taeda. Cerne, Lavras, v. 7, n. 1, p. 20 - 40, 2001.

ASSIS, A. L. de; SCOLFORO, J. R. S.; MELLO, J. M. de; OLIVEIRA, A. D. de. Avaliação de modelos polinomiais não segmentados na estimativa de diâmetros e volumes comerciais de Pinus taeda. Ciência Florestal, Santa Maria, v. 12, n. 1, p. 89 - 107, 2002.

ASSMANN, E. The principles of forest yield study: studies in the organic production, structure, increment and yield of forest stands. Oxford: Pergamon Press, 1970. 506 p.

BURKHART, H. E.; TOMÉ, M. Modeling forest trees and stands. New York: Springer, 2012. 457 p.

DRESCHER, R.; SCHNEIDER, P. R.; FINGER, C. A. G. Sortimentos de Pinus elliottii Engelm para a região da Serra do Sudeste do estado do Rio Grande do Sul, Brasil. Ciência Florestal, Santa Maria, v. 9, n. 2, p. $55-73,1999$.

EISFELD, R. L.; MELlO, A. A.; SANQUETTA, C. R.; WEBER, K. S. Avaliação de modelos polinomiais na estimativa de volume total e por sortimento de Pinus taeda. Brasil Florestal, Brasília, v. 23, n. 79 , p. 9 - 15, 2004.

FAVALESSA, C. M. C.; UBIALLI, J. A.; CALDEIRA, S. F.; DRESCHER, R. Funções de afilamento não segmentadas e segmentadas para Tectona grandis na região centro-sul mato-grossense. Pesquisa Florestal Brasileira, Colombo, v. 32, n. 72, p. 373 - 387, 2012a.

FAVAlessa, C. M. C.; UBIAlli, J. A.; CALDEIRA, S. F.; DRESCHER, R.; ACOSTA, F. C. Equações de sortimentos para Tectona grandis na região centro-sul de Mato Grosso. Pesquisa Florestal Brasileira, Colombo, v. 32, n. 72, p. 389 - 399, 2012b.

FISCHER, F.; SCOLFORO, J. R. S.; ACERBI JÚNIOR, F. W.; MELLO, J. M. de; MAESTRI, R. Exatidão dos modelos polinomiais não segmentados e das razões entre volumes para representar o perfil do tronco de Pinus taeda. Ciência Florestal, Santa Maria, v. 11, n. 1, p. 167 - 188, 2001.

FLORIANO, E. P. Efeito da desrama sobre o crescimento e a forma de Pinus elliottii Engelm. 93 f. Dissertação (Mestrado em Engenharia Florestal) - Universidade Federal de Santa Maria, Santa Maria, 2004. 
FRIEDL, R. A. Dinâmica e prognose da forma dos fustes em povoamentos plantados de Araucaria angustifolia (Bert.) O. Ktze. 167 f. Dissertação (Mestrado em Engenharia Florestal) - Universidade Federal do Paraná, Curitiba, 1989.

LARSON, P. R. Stem form development of forest trees. Forest Science, Bethesda, Monograph 5, 1963. $42 \mathrm{p}$.

MACHADO, S. A.; FIGUEIREDO FILHO, A. Dendrometria. 2. ed. Guarapuava: UNICENTRO, 2009. $316 \mathrm{p}$.

MACHADO, S. A.; URBANO, E.; CONCEIÇÃO, M. B. da; FIGUEIREDO FILHO, A.; FIGUEIREDO, D. J. de. Comparação de modelos de afilamento do tronco para diferentes idades e regimes de desbaste em plantações de Pinus oocarpa Schiede. Boletim de Pesquisa Florestal, Colombo, n. 48, p. 41 - 64, 2004.

MIGUEL, E. P.; MACHADO, S. A.; FIGUEIREDO FILHO, A.; ARCE, J. E. Modelos polinomiais para representar o perfil e o volume do fuste de Eucalyptus urophylla na região norte do estado de Goiás. Floresta, Curitiba, v. 41, n. 2, p. 355 - 368, 2011.

MONTGOMERY, D. C.; PECK, E. A.; VINING, G. G. Introduction to linear regression analysis. 4. ed. New York: John Wiley \& Sons, 2006. 612 p.

PRATES, A. M. M.; MANZOLLI, J. I.; MIRA, M. A. F. B. Geografia física de Santa Catarina. Florianópolis: Editora Lunardelli, 1989. 112 p.

PRODAN, M.; PETERS, R.; COX, F.; REAL, P. Mensura Forestal. San José: Instituto Interamericano de Cooperación para la Agricultura (IICA), 1997. 586 p.

QUEIROZ, D. Avaliação de funções de afilamento e sortimentos para Mimosa scabrella Bentham em povoamentos nativos da região metropolitana de Curitiba/PR. $128 \mathrm{f}$. Dissertação (Mestrado em Ciências Florestais) - Universidade Federal do Paraná, Curitiba, 2006.

QUEIROZ, D.; MACHADO, S. A.; FIGUEIREDO FILHO, A.; ARCE, J. E.; KOEHLER, H. S. Avaliação e validação de funções de afilamento para Mimosa scabrella Bentham em povoamentos da região metropolitana de Curitiba/PR. Floresta, Curitiba, v. 36, n. 2, p. 183 - 199, 2006.

SCHNEIDER, P. R.; SCHNEIDER, P. S. P. Introdução ao manejo florestal. Santa Maria: FACOS UFSM, 2008. $566 \mathrm{p}$.

SCOLFORO, J. R. S. Mensuração florestal 3: Relações quantitativas em volume, peso e a relação hipsométrica. Lavras: ESAL/FAEPE, 1993. 292 p.

SCOLFORO, J. R. S.; RIOS, M. S.; OLIVEIRA, A. D. de; MELlO, J. M. de; MAESTRI, R. Acuracidade de equações de afilamento para representar o perfil do fuste de Pinus elliottii. Cerne, Lavras, v. 4, n. 1, p. 100 - 122, 1998.

SOUZA, C. A. M. de; CHASSOT, T.; FINGER, C. A. G.; SCHNEIDER, P. R.; FLEIG, F. D. Modelos de afilamento para o sortimento do fuste de Pinus taeda L. Ciência Rural, Santa Maria, v. 38, n. 9, p. 2506 $2511,2008$.

TONG, Q. J.; ZHANG, S. Y. Stem form variations in the natural stands of major commercial softwoods in eastern Canada. Forest Ecology and Management, Amsterdam, v. 256, n. 6, p. 1303 - 1310, 2008.

YOSHITANI JÚNIOR, M.; NAKAJIMA, N. Y.; ARCE, J. E.; MACHADO, S. A.; DRUSZCZ, J. P.; HOSOKAWA, R. T.; MELLO, A. A. de. Funções de afilamento para plantios desbastados de Pinus taeda. Floresta, Curitiba, v. 42, n. 1, p. 169 - 176, 2012. 
